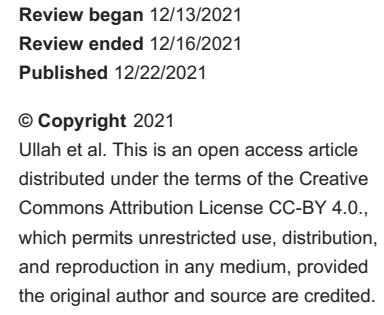

\section{Frequency of Atrial Fibrillation in Patients Presenting With Decompensated Heart Failure}

\author{
Rafi Ullah ${ }^{1}$, Ahmad Shiraz ${ }^{2}$, Sher Bahadur ${ }^{3}$, Farhat Shireen ${ }^{1}$ \\ 1. Cardiology, Lady Reading Hospital Peshawar, Peshawar, PAK 2. General Surgery, Hayatabad Medical Complex \\ Peshawar, Peshawar, PAK 3. Epidemiology and Public Health, Khyber Institute of Child Health, Peshawar, PAK
}

Corresponding author: Rafi Ullah, doctor_rafi@yahoo.com

\section{Abstract \\ Background}

Atrial fibrillation (AF) is a common concern in patients with heart disease, especially those with acute decompensated heart failure (ADHF). We conducted a cross-sectional study to determine the frequency of $\mathrm{AF}$ and associated risk factors among patients with ADHF at a tertiary care hospital in Peshawar, Pakistan.

\section{Methods}

We conducted a cross-sectional analytical study of hospitalized patients with ADHF treated in a tertiary care hospital in Peshawar, Pakistan, from June 5 to October 30, 2021. The study's primary outcome was the proportion of patients with ADHF who had AF, and our secondary outcome was examining the risk factors for AF. The College of Physicians and Surgeons Pakistan provided ethical approval of the study design. Data were analyzed using IBM SPSS Statistics for Windows version 24.0 (IBM Corp., Armonk, NY, USA). We applied the chi-square test to compare the proportion of AF concerning risk factors (i.e., comorbidities).

\section{Results}

One hundred ninety-four patients with ADHF were included in the study; 54.6\% were male and $45.4 \%$ female. Most (56.7\%) were older than 60, and 38.1\% were aged 40-60. The prevalence of AF was $38.1 \%$. Diabetes, hypertension, previous stroke, myocardial infarction (MI), and chronic obstructive pulmonary disease (COPD) were the most common comorbidities. All patients with ADHF with AF also had MI and hypertension. Patients of known coronary artery disease (CAD) but without MI, previous percutaneous coronary intervention $(\mathrm{PCI})$, or coronary artery bypass graft $(\mathrm{CABG})$ surgery were less associated with $\mathrm{AF}$ than other comorbidities.

\section{Conclusions}

We conducted this study to determine the incidence of AF among patients with ADHF. AF occurs in a significant amount of patients with ADHF, and the risk factors associated with AF in these patients include hypertension, history of MI, diabetes, and COPD. Healthcare professionals should screen patients with $\mathrm{ADHF}$ for $\mathrm{AF}$, especially those with common risk factors.

Categories: Cardiology, Epidemiology/Public Health

Keywords: comorbidities, risk factors, heart failure, hypertension, atrial fibrillation

\section{Introduction}

Atrial fibrillation (AF) is a common concern for patients with cardiac disease, especially those with arrhythmia and acute decompensated heart failure (ADHF) [1,2]. Approximately $20 \%-35 \%$ of admitted patients with ADHF have AF, even in the early stage of the disease [3]. While a high frequency of AF concurrent with ADHF has been reported, significant regional differences exist. In Pakistan, this issue has not been prioritized in previous research. The risk factors and adverse effects of AF in patients with heart failure vary from person to person and region to region because of differences in lifestyle, comorbidities, healthcare facilities, and treatment compliance. Therefore, we conducted this study to determine the frequency of AF and associated risk factors among patients with ADHF at a tertiary care hospital in Peshawar, Pakistan.

\section{Materials And Methods}

This hospital-based, cross-sectional study was conducted in a public sector teaching hospital in Peshawar, Pakistan, from June 5 to October 30, 2021, using consecutive sampling. Ethical approval was granted by the College of Physicians and Surgeons Pakistan on June 4, 2021. The study included men and women older than age 18 who presented with ADHF to the hospital as confirmed by echocardiogram. We excluded patients with a history of rheumatic heart disease and congenital abnormalities (also confirmed by echocardiogram). All study participants provided written informed consent to be included. All patients with ADHF were assessed 


\section{Cureus}

for the presence of AF based on an electrocardiogram. We obtained detailed clinical history for known comorbidities. We also evaluated previous investigations and prescriptions for all participants. According to the official data, approximately 600 patients of ADHF presented annually, so assuming $20 \%$ anticipated population [3], 0.05\% precision, and 95\% confidence level and considering $10 \%$ loss to follow-up, the expected sample size was 194.

We collected data using a structured proforma, transferred the information into a Microsoft Excel spreadsheet (Microsoft Inc., Redmond, WA, USA), and then analyzed using IBM SPSS Statistics for Windows version 24.0 (IBM Corp., Armonk, NY, USA). Descriptive statistics are presented for the proportions of comorbidities and coexistence of AF. We used the chi-square test to determine the associations between the risk factors for $\mathrm{AF}$ in patients with $\mathrm{ADHF}$ for comparative analysis. P-values less than 0.05 were considered statistically significant.

\section{Results}

A total of 194 patients were included in the study (106 men (54.6\%) and 88 women (45.4\%)). Most patients (n $=110(56.7 \%)$ ) were older than age 60, followed by aged $74(38.1 \%)$, aged 40-60 (10\%), and then aged 20-40 (5.2\%). AF was found in 38.1\% of patients with ADHF (Figure 1).

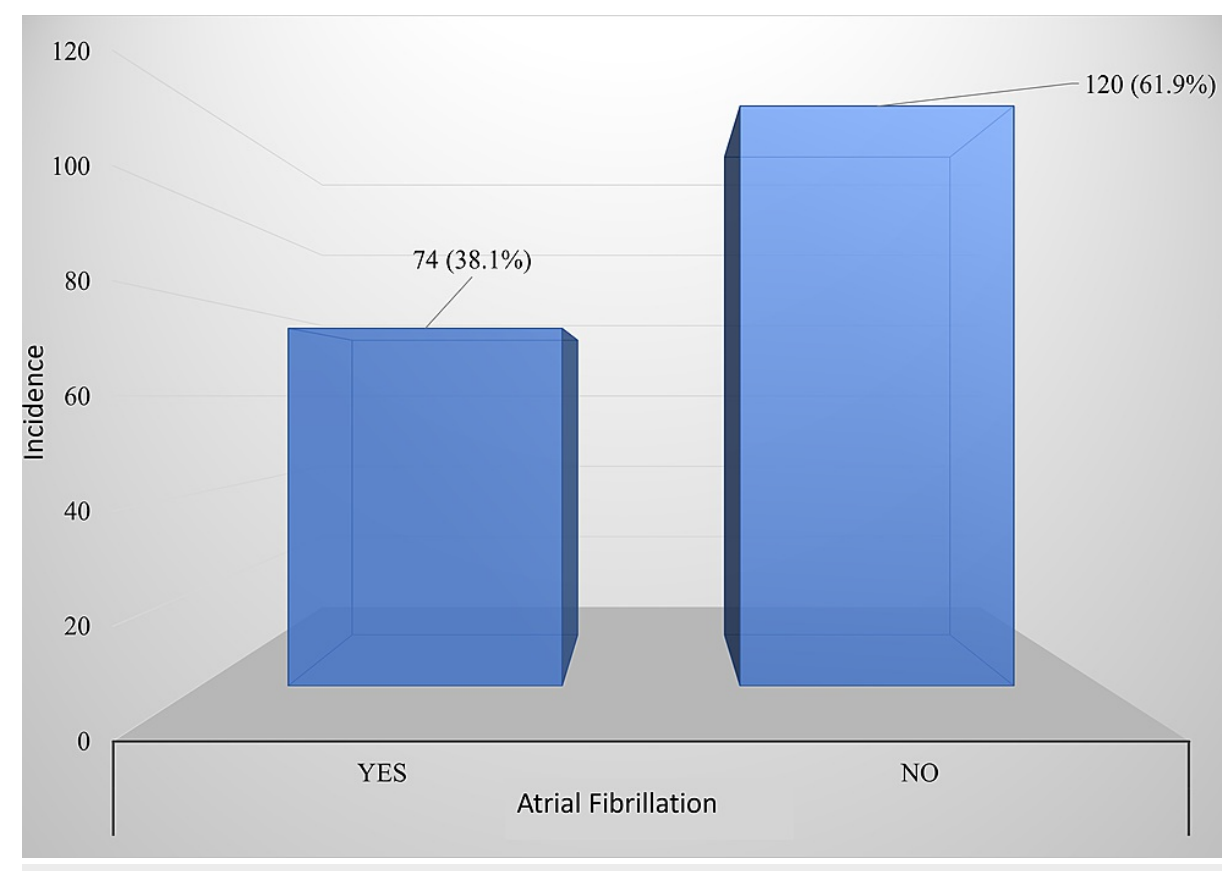

FIGURE 1: Frequency of AF among patients with ADHF

AF: atrial fibrillation; ADHF: acute decompensated heart failure

In our study, the most common comorbidities among patients with ADHF were myocardial infarction (MI) (n $=148(76.3 \%)$ ), hypertension $(n=101(52.1 \%))$, diabetes $(n=98(50.5 \%))$, chronic obstructive pulmonary disease (COPD) $(n=89(45.9 \%))$, coronary artery disease $(\mathrm{CAD})(\mathrm{n}=86(44.3 \%))$, stroke $(\mathrm{n}=82(42.3 \%))$, previous coronary artery bypass graft $(\mathrm{CABG})(\mathrm{n}=25(12.9 \%))$, and percutaneous coronary intervention (PCI) $(\mathrm{n}=24(12.4 \%))($ Table 1$)$. 


\section{Cureus}

\begin{tabular}{|l|llll|}
\hline Parameters & Status & & & \\
\\
CAD & Yes & $f(\%)$ & No & $f(\%)$ \\
History of MI & 86 & $(44.3)$ & 108 & $(55.7)$ \\
Previous PCI & 148 & $(76.3)$ & 46 & $(23.7)$ \\
Previous CABG & 24 & $(12.4)$ & 170 & $(87.6)$ \\
Diabetes & 25 & $(12.9)$ & 169 & $(87.1)$ \\
COPD & 98 & $(50.5)$ & 96 & $(49.5)$ \\
Hypertension & 89 & $(45.9)$ & 105 & $(54.1)$ \\
Previous stroke/TIA & 101 & $(52.1)$ & 93 & $(47.9)$ \\
\hline
\end{tabular}

\section{TABLE 1: Common comorbidities associated with ADHF}

ADHF: acute decompensated heart failure; CAD: coronary artery disease; MI: myocardial infarction; $\mathrm{PCl}$ : percutaneous coronary intervention; CABG: coronary artery bypass graft; COPD: chronic obstructive pulmonary disease; TIA: transient ischemic attack

Patients with concurrent AF and ADHF were significantly positive for a history of MI, CAD, diabetes, hypertension, and previous stroke/transient ischemic attack (TIA), but sex had no significant association with AF concurrent with ADHF ( $p=0.49)$. In patients with AF, $57(77 \%)$ had a history of CAD, $66(89.2 \%)$ were positive for a history of MI, and $49(66.2 \%)$ had diabetes. Hypertension was the leading comorbidity $(\mathrm{n}=$ $53(71.6 \%))$, followed by COPD $(\mathrm{n}=48(64.9 \%))$, and previous stroke/TIA $(\mathrm{n}=44(59.5 \%))$. Only $14(18.9 \%)$ patients had a history of PCI, and 18 (24.3\%) had previous CABG. The differences for all these parameters were significant $(\mathrm{p}<0.001)$ (Table 2). 


\section{Cureus}

\begin{tabular}{|c|c|c|c|c|}
\hline \multirow[t]{2}{*}{ Parameters/comorbidities } & & \multicolumn{2}{|l|}{ Atrial fibrillation } & \multirow[b]{2}{*}{ P-value } \\
\hline & & ADHF with $A F(n=74)$ & ADHF without AF $(n=120)$ & \\
\hline \multirow[t]{2}{*}{ Gender } & Male & $45(60.8 \%)$ & $67(55.8 \%)$ & 0.49 \\
\hline & Female & $29(39.2 \%)$ & $53(44.3 \%)$ & \\
\hline \multirow[t]{2}{*}{ CAD } & Yes & $57(77 \%)$ & $29(24.2 \%)$ & 0.0001 \\
\hline & No & $17(23 \%)$ & $91(75.8 \%)$ & \\
\hline \multirow[t]{2}{*}{ History of MI } & Yes & $66(89.2 \%)$ & $82(68.3 \%)$ & 0.001 \\
\hline & No & $8(10.8 \%)$ & $38(31.7 \%)$ & \\
\hline \multirow[t]{2}{*}{ Previous $\mathrm{PCl}$} & Yes & $14(18.9 \%)$ & $10(8.3 \%)$ & 0.03 \\
\hline & No & $60(81.1 \%)$ & $110(91.7 \%)$ & \\
\hline \multirow[t]{2}{*}{ Previous CABG } & Yes & $18(24.3 \%)$ & $7(5.8 \%)$ & 0.0001 \\
\hline & No & $56(75.7 \%)$ & $113(94.2 \%)$ & \\
\hline \multirow[t]{2}{*}{ Diabetes mellitus } & Yes & $49(66.2 \%)$ & 49 (40.8\%) & 0.001 \\
\hline & No & $25(33.8 \%)$ & $71(59.2 \%)$ & \\
\hline \multirow[t]{2}{*}{ COPD } & Yes & $48(64.9 \%)$ & $41(34.2 \%)$ & 0.0001 \\
\hline & No & $26(35.1 \%)$ & $79(65.8 \%)$ & \\
\hline \multirow[t]{2}{*}{ Hypertension } & Yes & $53(71.6 \%)$ & $48(40 \%)$ & 0.0001 \\
\hline & No & $21(28.4 \%)$ & $72(60 \%)$ & \\
\hline \multirow[t]{2}{*}{ Previous stroke/TIA } & Yes & $44(59.5 \%)$ & $38(31.7 \%)$ & 0.0001 \\
\hline & No & $30(40.5 \%)$ & 82 (68.3\%) & \\
\hline
\end{tabular}

\section{TABLE 2: Comparison of risk factors among patients ADHF with and without AF}

ADHF: acute decompensated heart failure; AF: atrial fibrillation; CAD: coronary artery disease; MI: myocardial infarction; PCl: percutaneous coronary intervention; CABG: coronary artery bypass graft; COPD: chronic obstructive pulmonary disease; TIA: transient ischemic attack

\section{Discussion}

AF in patients with ADHF is relatively common and has a poor prognosis, but its prevalence and risk factors seem to vary by country [4]. Therefore, this study sought to determine the frequency of AF among patients with ADHF in Pakistan. Our results indicate that the prevalence of AF among patients with ADHF was $38.1 \%$, which is supported by data from Turkey, where $39 \%$ of patients with ADHF had a history of AF [5]. However, 25.4\% of the subjects had AF in Cameroon [6]. In Brazil, a review of five years of hospital records found that $40 \%$ of patients with $\mathrm{ADHF}$ had $\mathrm{AF}$ [3]. In another study, approximately $20 \%-35 \%$ of patients diagnosed with $\mathrm{ADHF}$ had $\mathrm{AF}$ at the time of admission, and one in three cases of AF were acute [7].

Also, AF was positively associated with advanced age (26\% for men and $23 \%$ for women older than age 40 [8]), ischemia, right ventricular (RV) dysfunctions, high ejection fraction (EF), and cardiomegaly, especially an enlarged left atrium $(\mathrm{p}<0.05)$ [1,3]. Additional risk factors reported by the literature include a history of hypertension, diabetes, MI, medications, socioeconomic status, and body mass index [9,10].

AF can lead to decompensation, which may act as a primary stimulus to acute heart failure. Because of this decompensation, there is an increase in left atrial pressure and decreased stroke volume. Patients with $\mathrm{AF}$ and ADHF frequently present with mitral valve regurgitation and have a poor prognosis with a high risk of stroke and increased risk of death [11,12]. Mortality was high for patients hospitalized more than once for AF and ADHF [13]. According to the literature, 39\% of patients with ADHF have a positive history of AF and were often in advanced age $[1,2,5]$. AF in ADHF was associated with stroke, increased blood pressure, and valvular diseases [5]. These changes make AF resistant to treatment using rhythm-controlled strategies [14]. Another study in Pakistan regarding the risk factors for AF reported that $55.3 \%$ of patients had a history of rheumatic heart disease, $34 \%$ had ischemia, $28 \%$ presented with RV dysfunction, and $34.5 \%$ had mitral valve regurgitation [10]. 
Heart failure incidence is associated with the time of heart failure onset. One study reported that at the acute stage, the incidence of $\mathrm{AF}$ is $10.3 \%$, and the incidence rate was $27.3 / 1000$ person-years [15]. A $75 \%$ increase in the occurrence of AF takes place in the first six months of heart failure (odds ratio: 3.6). This suggests that AF is high in the initial phase of HF, particularly within six months of onset [15].

MI was the most common comorbidity in our study population (77.3\%), followed by hypertension (52.1\%) and diabetes (50.5\%). CAD (44.3\%) and stroke (42.3\%) were also present, but only a few instances of CABG (12.9\%) and PCI (12.4\%) were noted. Our findings were consistent with other reports where CAD was the second leading comorbidity after hypertension among patients with ADHF. One study reported that hypertension (70\%) and CAD (60\%) were present in patients with $\mathrm{ADHF}$, followed by valvular problems (44\%), diabetes (40\%), cardiomyopathy (25\%), and renal impairment (20\%) [16]. Another study reported a positive association between AF and advanced age $(p<0.0001)$ [3]. In that study, AF was also common in patients with RV dysfunction, left atrium enlargement, hypertension, low EF, and nonischemic heart disease. AF was also associated with increased hospital stay and high in-hospital mortality [3].

Comorbidities such as MI, hypertension, CAD, diabetes, and COPD were strongly associated with AF (p < 0.001). However, AF was less common among patients with a previous history of stroke, CABG, or PCI. Other studies have confirmed that these comorbidities are associated with adverse clinical outcomes due to atrial stiffness, vasoconstrictions, and inflammation [17,18]. Kazeminia et al. reported that diabetes was the most common comorbidity among patients with heart failure, and the prevalence increases over time [19]. Diabetes is associated with half of the mortality among patients with ADHF [20]. Joseph et al. found that $70 \%$ of patients with ADHF had hypertension, $60 \%$ had CAD, and $40 \%$ had diabetes [16].

Patients with AF and ADHF had more frequent rehospitalization rates and longer hospital stays than patients without AF [21-23]. This indicates that apart from structural abnormalities, there are several other risk factors that significantly contribute to the development of AF and adverse clinical outcomes.

\section{Limitations}

Our study was limited to a single-center study of one tertiary care hospital due to a shortage of time. The study examined a limited number of risk factors due to limited funding. Future research would benefit from multicenter research with a broader range of risk factors studied.

\section{Conclusions}

We conducted this study to determine the prevalence of AF among patients with ADHF in Pakistan. AF occurs in a significant amount of patients with ADHF, and the risk factors associated with AF in these patients include hypertension, history of MI, diabetes, and COPD. These risk factors with both AF and ADHF can lead to poor patient outcomes. Healthcare professionals should screen patients with ADHF for AF, especially in the presence of common risk factors.

\section{Additional Information \\ Disclosures}

Human subjects: Consent was obtained or waived by all participants in this study. Animal subjects: All authors have confirmed that this study did not involve animal subjects or tissue. Conflicts of interest: In compliance with the ICMJE uniform disclosure form, all authors declare the following: Payment/services info: All authors have declared that no financial support was received from any organization for the submitted work. Financial relationships: All authors have declared that they have no financial relationships at present or within the previous three years with any organizations that might have an interest in the submitted work. Other relationships: All authors have declared that there are no other relationships or activities that could appear to have influenced the submitted work.

\section{References}

1. Kiuchi K, Shirakabe A, Kobayashi N, et al.: Prognostic impact of new-onset atrial fibrillation associated with worsening heart failure in aging patients with severely decompensated acute heart failure. Int J Cardiol. 2020, 302:88-94. 10.1016/j.ijcard.2019.09.020

2. Iwahashi N, Takahashi H, Abe T, et al.: Urgent control of rapid atrial fibrillation by landiolol in patients with acute decompensated heart failure with severely reduced ejection fraction. Circ Rep. 2019, 1:422-30. 10.1253/circrep.CR-19-0076

3. Mendes Fde S, Atié J, Garcia MI, Gripp Ede A, Sousa AS, Feijó LA, Xavier SS: Atrial fibrillation in decompensated heart failure: associated factors and in-hospital outcome. Arq Bras Cardiol. 2014, 103:31522. $10.5935 / \mathrm{abc} .20140123$

4. Jobs A, Schwind J, Katalinic A, et al.: Prognostic significance of atrial fibrillation in acute decompensated heart failure with reduced versus preserved ejection fraction. Clin Res Cardiol. 2019, 108:74-82.

5. Kocabaş U, Sinan ÜY, Aruğaslan E, et al.: Clinical characteristics and in-hospital outcomes of acute decompensated heart failure patients with and without atrial fibrillation. Anatol J Cardiol. 2020, 23:260-7. 10.14744/AnatolJCardiol.2020.94884

6. Lemogoum D, Kamdem F, Ba H, et al.: Epidemiology of acutely decompensated systolic heart failure over 
the 2003-2013 decade in Douala General Hospital, Cameroon. ESC Heart Fail. 2021, 8:481-8. 10.1002/ehf2.13098

7. DiMarco JP: Atrial fibrillation and acute decompensated heart failure . Circ Heart Fail. 2009, 2:72-3. 10.1161/CIRCHEARTFAILURE.108.830349

8. Lubitz SA, Benjamin EJ, Ellinor PT: Atrial fibrillation in congestive heart failure . Heart Fail Clin. 2010, 6:187-200. 10.1016/j.hfc.2009.11.001

9. Schnabel RB, Sullivan LM, Levy D, et al.: Development of a risk score for atrial fibrillation (Framingham Heart Study): a community-based cohort study. Lancet. 2009, 373:739-45. 10.1016/S0140-6736(09)60443-8

10. Sarfraz A, Akhtar P, Hassan Rizvi SN, Karim M: Risk factors of atrial fibrillation in patients with heart failure. Cureus. 2018, 10:e3774. 10.7759/cureus.3774

11. Hirai K, Kawakami R, Nogi M, et al.: Impact of atrial fibrillation on the prognosis of acute decompensated heart failure with and without mitral regurgitation. Circ Rep. 2021, 3:388-95. 10.1253/circrep.CR-21-0027

12. Ivănescu AC, Dan GA: Stroke risk scores to predict hospitalization for acute decompensated heart failure in atrial fibrillation patients. Rom J Intern Med. 2021, 59:73-82. 10.2478/rjim-2020-0032

13. Kamioka M, Yoshihisa A, Nodera M, et al.: The clinical implication of new-onset in-hospital atrial fibrillation in patients with acute decompensated heart failure. J Arrhythm. 2020, 36:874-82. 10.1002/joa3.12386

14. Mladěnka P, Applová L, Patočka J, et al.: Comprehensive review of cardiovascular toxicity of drugs and related agents. Med Res Rev. 2018, 38:1332-403. 10.1002/med.21476

15. Martín-Pérez M, Ruigómez A, Michel A, García Rodríguez LA: Incidence and risk factors for atrial fibrillation in patients with newly diagnosed heart failure. J Cardiovasc Med (Hagerstown). 2016, 17:608-15. 10.2459/JCM.0000000000000403

16. Joseph SM, Cedars AM, Ewald GA, Geltman EM, Mann DL: Acute decompensated heart failure: contemporary medical management. Tex Heart Inst J. 2009, 36:510-20.

17. Rabe KF, Hurst JR, Suissa S: Cardiovascular disease and COPD: dangerous liaisons? . Eur Respir Rev. 2018, 27:10.1183/16000617.0057-2018

18. Huang Q, Xiong H, Shuai T, et al.: Risk factors for new-onset atrial fibrillation in patients with chronic obstructive pulmonary disease: a systematic review and meta-analysis. PeerJ. 2020, 8:e10376. 10.7717/peerj.10376

19. Kazeminia M, Salari N, Mohammadi M: Prevalence of cardiovascular disease in patients with type 2 diabetes mellitus in Iran: a systematic review and meta-analysis. J Diabetes Res. 2020, 2020:3069867. $10.1155 / 2020 / 3069867$

20. Einarson TR, Acs A, Ludwig C, Panton UH: Prevalence of cardiovascular disease in type 2 diabetes: a systematic literature review of scientific evidence from across the world in 2007-2017. Cardiovasc Diabetol. 2018, $17: 83.10 .1186 /$ s12933-018-0728-6

21. Teerlink JR, Alburikan K, Metra M, Rodgers JE: Acute decompensated heart failure update . Curr Cardiol Rev. 2015, 11:53-62. 10.2174/1573403x09666131117174414

22. Darden D, Nishimura M, Sharim J, Maisel A: An update on the use and discovery of prognostic biomarkers in acute decompensated heart failure. Expert Rev Mol Diagn. 2019, 19:1019-29. 10.1080/14737159.2019.1671188

23. Abualnaja S, Podder M, Hernandez AF, et al.: Acute heart failure and atrial fibrillation: insights from the Acute Study of Clinical Effectiveness of Nesiritide in Decompensated Heart Failure (ASCEND-HF) trial. J Am Heart Assoc. 2015, 4:e002092. 10.1161/JAHA.115.002092 